\title{
Lung Clearance Index in Children with Sickle Cell Disease
}

\author{
Evans Machogu ${ }^{1}$, Monica Khurana ${ }^{1}$, Jennifer Kaericher ${ }^{1}$, Charles Clem ${ }^{2}$, James Slaven ${ }^{1}$, \\ Joseph Hatch ${ }^{3}$, Stephanie Davis ${ }^{4}$, and Stacey Peterson-Carmichael ${ }^{5}$ \\ ${ }^{1}$ Indiana University School of Medicine \\ ${ }^{2}$ James Whitcomb Riley Hospital for Children \\ ${ }^{3}$ University of North Carolina System \\ ${ }^{4}$ University of North Carolina at Chapel Hill School of Medicine \\ ${ }^{5}$ Wake Forest University
}

July 7, 2020

\begin{abstract}
Introduction: The Lung Clearance Index (LCI) derived from the multiple-breath washout test (MBW), is both feasible and sensitive to early lung disease detection in young children with cystic fibrosis and asthma. The utility of LCI has not been studied in children with sickle cell disease (SCD). We hypothesized that children with SCD, with or without asthma or airway hyper reactivity (AHR), would have an elevated LCI compared to healthy controls. Methods: Children with SCD from a single center between the ages of 6-18 years were studied at baseline health and completed MBW, spirometry, plethysmography and blood draws for serum markers. Results were compared to healthy controls of similar race, age and gender. Results: Control subjects $(\mathrm{n}=35)$ had a significantly higher daytime oxygen saturation level, weight and body mass index (BMI) but not height compared to subjects with SCD ( $\mathrm{n}=34)$. Total Lung Capacity(TLC) z-scores were significantly higher in the healthy controls compared to those with SCD $(0.87$ (1.13), $0.02(1.27), \mathrm{p}=0.005)$ while differences in Forced Expiratory Volume in 1 second (FEV1) z-scores approached significance $(0.26(0.97),-0.22(1.09), \mathrm{p}=0.055)$. There was no significant difference in LCI among the comparison groups $(7.29(0.72), 7.40(0.69), \mathrm{p}=0.514)$. Conclusion: LCI did not differentiate SCD from healthy controls in children between the ages of 6 and 18 years at baseline health. TLC may be an important pulmonary function measure to follow longitudinally in the pediatric SCD population.
\end{abstract}

\section{INTRODUCTION}

Sickle Cell Disease (SCD) affects about one in every 400 African-Americans in the United States ${ }^{1}$ with significant complications including recurrent severe pain episodes ${ }^{2}$ and acute chest syndrome (ACS) ${ }^{3}$ for which each episode carries a $4 \%$ chance of death ${ }^{4}$. Pulmonary complications are the cause of death in over $50 \%$ of autopsies in SCD patients yet reliable and sensitive tests to detect early lung disease are not available. An estimated 30-70\% of children with SCD have wheezing and airway hyper-reactivity (AHR) and air trapping ${ }^{5-11}$ and those with asthma have higher rates of pain and ACS as well as premature death ${ }^{12-17}$. Ultimately, patients with multiple episodes of ACS and wheezing develop Sickle Cell Lung Disease (SCLD), which is a restrictive lung disorder ${ }^{18}$, often associated with pulmonary hypertension.

Diagnosis of early lung disease in SCD is delayed as spirometry and plethysmography, measures of obstructive or restrictive lung disease, cannot often be reliably performed in children under the age of 5 years. Additionally, spirometry is relatively insensitive in identifying and assessing early lung disease in the smaller, more distal airways until significant disease has developed ${ }^{19,20}$. The multiple breath washout (MBW) technique, which measures the Lung Clearance Index (LCI), is a novel noninvasive measurement of ventilation inhomogeneity that is simple, involves no risk or hazardous exposures and can be used in children, including preschoolers. LCI has been shown to be a sensitive tool in detecting lung disease in cystic fibrosis and asthma 
before any changes are apparent on spirometry ${ }^{21,22}$ and has been demonstrated to be abnormal in patients with normal lung function on spirometry ${ }^{23,24}$.

To date, MBW has not been studied in children with SCD. One study explored the potential relationship between nitrogen single-breath washout (N2SBW) and exercise intolerance in 38 adults with SCD (median age of 28 years) ${ }^{25}$. Results suggested that phase III slope (SIIIN2) values correlated with hemoglobin level, forced vital capacity (FVC), diffusing capacity and $\mathrm{SpO} 2$ after exercise. Given this, assessing the ability of MBW to detect potential ventilation inhomogeneity may prove beneficial and allow for earlier intervention in patients where the disease may go unrecognized. Since pulmonary complications such as ACS, wheezing and airway hyper-reactivity (AHR) often start early in $\mathrm{SCD}^{11,26}$ we hypothesized that children with SCD (with or without asthma) would have an abnormal and elevated LCI compared to healthy controls. Findings of abnormal LCI in children with SCD during which time spirometry may be normal would be useful in initiating early intervention and more in-depth diagnostic screening.

\section{METHODS}

In this case-control study, we recruited patients with SCD with or without a physician diagnosis of asthma and healthy African American controls between 6 and 18 years of age. Inclusion/exclusion criteria: Inclusion criteria were a diagnosis of sickle cell disease (HbSS, HbS $\beta$-thalassemia ${ }^{+}, \mathrm{HbS} \beta$-thalassemia ${ }^{0}, \mathrm{HbSC}$, HbSO Arab) confirmed by hemoglobin analysis. Age criteria was 6 to 18 years of age. Exclusion criteria were preterm birth (born at $<37$-week gestation) or an inability to complete study activities. Participants completed study activities only if they were at baseline health without respiratory or SCD related complications occurring within the previous 4 weeks. Healthy African American controls between the ages of 6 to 18 years were also recruited but were excluded from the study for a diagnosis of asthma, any episodes of wheezing leading to shortness of breath in the previous 12 months or presence of a bronchodilator response ([?] $12 \%$ increase in $\mathrm{FEV}_{1}$ or FVC post-bronchodilator).

This study was approved by our institutional IRB and informed consent and assent, where applicable, were obtained for all participants. A thorough history was obtained, and a physical examination completed at the time of study participation. A review of the participants' medical charts was conducted to obtain data regarding episodes of ACS, severe pain crises requiring an inpatient stay, a physician-documented diagnosis of asthma and other co-morbidities. All participants performed pulmonary function tests (PFT) including MBW (Eco Medics Exhalyzer D, software version 3.1.6) using nitrogen as the washout gas, plethysmography (Morgan Scientific, ComPAS software version 1.10.2642.4440), and pre- and post- bronchodilator spirometry (Morgan Scientific, ComPAS software version 1.10.2642.4440). Blood was obtained from healthy controls at the time of the study visit as well as in those SCD subjects with no laboratory studies in the previous 3 months

\section{Study protocol and procedures:}

The study participants first completed MBW according to American Thoracic Society (ATS) guidelines ${ }^{27}$. LCI was determined according to standardized protocols ${ }^{28}$. Subsequently spirometry followed by lung volumes (plethysmography) were performed according to ATS criteria and standards ${ }^{29,30}$. To measure airway hyper-responsiveness ${ }^{27}$ (AHR), spirometry was repeated 20 minutes after administration of 4 puffs of albuterol via MDI. For healthy controls and SCD patients without a recent blood draw (3 months), whole blood samples were collected for processing on the day of testing.

Definitions: Obstructive lung disease was defined as 1 or more lung function measures $\left(\mathrm{FEV}_{1}\right.$ or $\mathrm{FEV}_{1} / \mathrm{FVC}$ ) < lower limit of normal (LLN) equivalent to a z-score of -1.645 based on Global Lung Initiative (GLI) normative data for age, height, weight and race. Restrictive lung disease was defined as Total Lung Capacity (TLC) or Forced Vital Capacity (FVC) <LLN based on GLI normative data for age, height, weight and race. $A H R$ was defined as having a positive bronchodilator response after repeat spirometric measures with an increase in $\mathrm{FEV}_{1}$ and/or FVC of [?]12\% from baseline ${ }^{31}$.

Statistical Analysis: The sample size was based on results from Zwitserloot et al. ${ }^{32}$ where the comparison 
of LCI between patients with asthma and healthy children (controls) showed a LCI difference of 0.27 with standard deviations of 0.48 and 0.38 for the two groups respectively. There are no defined normative LCI values in African American children. We assumed a similar LCI difference and standard deviation between SCD and healthy control children. In order to achieve $80 \%$ power and a $5 \%$ error rate, we needed to enroll 42 children in each group. Demographics, clinical events, lab results, and pulmonary function data were compared across groups using Student's t-tests for continuous variables. Wilcoxon tests were performed for non-linear data. Chi-Square tests were performed for categorical variables and Fisher's Exact tests were used when cell counts were low. Correlation analyses were performed to analyze the associations between clinical and pulmonary function data. To check for possible moderating effects due to participant age, interaction terms were assessed with age groups 6-12 years of age and 13-18 years, as well as performing analyses stratified by age groups. Multivariable models were then used to determine if associations with LCI would become attenuated in the larger model. Variables in the multivariable model were chosen due to statistical significance at the bivariate level and for clinical meaningfulness. All analytic assumptions were verified, with non-parametric tests (i.e. Wilcoxon tests and Spearman correlations) used when data were non-linear. Collinearity was assessed in the multivariable model with variance inflation factors. All analyses were performed using SAS v9.4 (SAS Institute, Cary, NC).

\section{RESULTS}

Out of a total of 193 subjects assessed for study eligibility, 69 completed all study requirements and are included in our analysis

\section{(Figure 1).}

Baseline demographics are shown in Table 1 . Thirty-four (34) participants with SCD and 35 healthy controls met criteria for analysis. We categorized the participants and controls into two age groups (6-12 years and 13-18 years). Control subjects had a significantly higher weight and body mass index (BMI) but not height compared to patients with SCD. Of the 34 participants with SCD, 27 had HbSS and HBS $\beta^{0}$ as their genotype and thus eligible for hydroxyurea at the time of study participation. Only 20 of the 27 patients eligible for hydroxyurea were on it at time of our study. Four participants required a NICU stay for various reasons other than respiratory distress though one was briefly placed on oxygen.

\section{Clinical and laboratory comparisons}

Four from the control group declined a blood draw (or had inadequate sample for processing) and were presumed to be either carriers or have HBAA genotype given no stigmata of SCD (Table 2). SCD subjects demonstrated a significantly lower hemoglobin concentration, higher white blood cell count and lower daytime oxygen saturation level.

A comparison of pulmonary function data is shown in table 3 . TLC and $\mathrm{FEV}_{1}$ were significantly higher in the healthy controls compared to those with SCD. There was no significant difference in LCI among the comparison groups. In our patient cohort, we saw no changes in LCI with increasing age (Figure 2 )

Amongst the subjects with SCD, a normal pattern of lung function was noted in 31 patients (91\%), an obstructive pattern in 3 patients $(9 \%)$, and a mixed obstructive/restrictive pattern in only 1 patient (3\%). Four (12\%) of the SCD participants demonstrated bronchodilator responsiveness. However, nineteen (56\%) had a physician diagnosis of asthma. There was no statistically significant difference in pulmonary function indices between patients with SCD $(n=18)$ and healthy controls $(n=17)$ ages 6-12 years. However, a comparison between participants with SCD and healthy controls ages 13-18 years revealed that TLC and FEV 1 z-score values were significantly higher in the healthy control group $(0.86+/-1.25$ vs $-0.22+/-1.37 ; \mathrm{P}=0.02)$ and $(0.28+/-1.11$ vs $-0.53+/-1.01 ; \mathrm{p}=0.03)$.

SCD subjects of all ages without a history of asthma had significantly higher TLC z-score values vs those with asthma $(0.74+/-1.21$ vs $-0.32+/-1.11$ with asthma; $\mathrm{p}=0.002)$ but there were no differences in LCI $(7.31+/-0.65$-without asthma vs $7.44+/-0.83$ with asthma; $\mathrm{p}=0.5085)$. 
A history of ACS was associated with a lower TLC z-score $(-0.74+/-0.96$ vs $0.41+/-1.30, \mathrm{p}=0.015)$ and $\mathrm{FEV}_{1}(-0.76+/-0.54,0.08+/-1.25 ; \mathrm{P}=0.013)$. However, a history of ACS was not associated with higher LCI ((7.25 (0.48) for history of ACS vs $7.47(0.78) ; \mathrm{p}=0.411))$. A history of asthma or response to bronchodilator was not significantly associated with LCI

\section{Correlations between LCI, spirometry and plethysmography}

Amongst all study participants, FVC, $\mathrm{FEV}_{1}$, RV and TLC are highly correlated with LCI, although these associations lose statistical significance when looking at only the sickle cell group with the only significant correlation being with TLC (table 4).

\section{Multivariate analysis}

In the adjusted multivariable model for the SCD group only(table 5) hemoglobin was independently associated with LCI when all the variables are included in the model.

\section{DISCUSSION}

To our knowledge, this is the first study to evaluate LCI in the childhood sickle cell population. In this study, LCI did not differentiate SCD from healthy controls in children between the ages of 6 and 18 years of age at baseline health. Furthermore, we saw no cross-sectional differences in LCI with increasing age. Additionally, neither a history of asthma nor ACS was associated with a significantly higher LCI among the SCD participants compared to healthy controls. There was a high correlation between $\mathrm{FEV}_{1}$, FVC, RV and TLC with LCI amongst all subjects, although some of these associations lose statistical significance when looking at only the sickle cell group likely due to small sample size.

In the SCD only group, the only significant association with LCI was TLC. There was no correlation between LCI values and past episodes of ACS or diagnosis of asthma in our study. TLC was significantly higher in healthy controls compared to SCD subjects and was highly correlated with LCI in the SCD group only. This may suggest that TLC is a much more sensitive measure of early lung disease in the adolescent group (ages 13-18yrs) before any obvious obstructive or restrictive patterns emerge. Future studies focusing on plethysmography may bring better understanding to the potential use of LCI for detecting lung disease in this population.

Our data support previous studies ${ }^{33,34}$ that have reported decreased baseline oxygen saturation in the SCD population. In children between the ages of 13 and 18 years of age, TLC and $\mathrm{FEV}_{1}$ were significantly higher in the healthy subjects compared to those with SCD. This would further support existing literature that lung disease in sickle cell patients worsens over time, and that identifying an early screening technique to differentiate the higher risk SCD populations before they have recurrent ACS and recurrent VOC is critical.

Three (9\%) of our patients demonstrated an obstructive disease pattern on spirometry and only 1 (3) \% had a mixed obstructive/restrictive disease pattern. This is similar to previous studies ${ }^{35}$ with the majority of SCD patients having normal lung function or some type of mixed picture. The number of SCD patients with asthma based on physician diagnosis was 19 (56\%). There was likely some recruitment bias as subjects who were approached for the study and agreed to participate were often patients seen in Pediatric Pulmonology clinic. LDH values differed significantly with a mean of 447 in the "asthma" group versus 306 in the "no asthma" group. These values may reflect treatment and adherence to hydroxyurea. Our data revealed that $80 \%$ of the SCD "asthma" group verbalized having hydroxyurea as a current prescription medication versus $6 \%$ in the "no asthma" group. This was not part of our aims or hypotheses but interesting for future research into potential protective effects of hydroxyurea in SCD patients with pulmonary diagnoses other than ACS such as asthma, wheezing or chronic hypoxia.

Study limitations include the inability to reach our goal enrollment of 42 participants in each of the SCD and control study groups. We were able to report data on 69 total participants, and due to the lack of normative LCI data for African American participants, our power calculation was performed based on LCI in pediatric asthma. However, our research allowed the opportunity to collect LCI data in an African 
American normative cohort. Pritchard et $\mathrm{al}^{36}$ studied the basic biologic processes behind lung disease in SCD animal models, however, the in-vivo pathophysiology of lung disease in SCD, especially in the "healthy" SCD pediatric population is still not well understood. The current understanding of pathophysiology in SCD led to the proposed hypothesis to test LCI where perhaps using other non-invasive methods such as FOT (forced oscillation technique) would be more appropriate. The other limitation is the nature of the study design such that the patients were not followed longitudinally to assess future frequency of ACS, and other SCD complications. Future studies in the use of LCI in the SCD population might focus on LCI values during and after acute complications such as ACS, wheezing episode, asthma exacerbation. The other population of interest would be the adult SCD group where the incidence of respiratory disease and abnormal PFT's is much higher.

In conclusion, there was no significant difference in LCI measures between SCD and healthy controls at baseline health. Furthermore, TLC may be an important pulmonary function measure to follow longitudinally in the pediatric SCD population. Future studies at time of ACS recovery, or in the adult population may bring better understanding to the potential use of LCI for detecting lung disease in this population.

\section{REFERENCES}

1. Ashley-Koch A, Yang Q, Olney RS. Sickle hemoglobin (HbS) allele and sickle cell disease: a HuGE review. American Journal of Epidemiology 2000;151:839-45.

2. Platt OS, Thorington BD, Brambilla DJ, et al. Pain in sickle cell disease. Rates and risk factors. N Engl J Med 1991;325:11-6.

3. Castro O, Brambilla DJ, Thorington B, et al. The acute chest syndrome in sickle cell disease: incidence and risk factors. The Cooperative Study of Sickle Cell Disease. Blood 1994;84:643-9.

4. Vichinsky EP, Neumayr LD, Earles AN, et al. Causes and outcomes of the acute chest syndrome in sickle cell disease. National Acute Chest Syndrome Study Group. N Engl J Med 2000;342:1855-65.

5. Koumbourlis AC, Zar HJ, Hurlet-Jensen A, Goldberg MR. Prevalence and reversibility of lower airway obstruction in children with sickle cell disease. The Journal of pediatrics 2001;138:188-92.

6. Leong MA, Dampier C, Varlotta L, Allen JL. Airway hyperreactivity in children with sickle cell disease. The Journal of pediatrics 1997;131:278-83.

7. Ozbek OY, Malbora B, Sen N, Yazici AC, Ozyurek E, Ozbek N. Airway hyperreactivity detected by methacholine challenge in children with sickle cell disease. Pediatric pulmonology 2007;42:1187-92.

8. Sen N, Kozanoglu I, Karatasli M, Ermis H, Boga C, Eyuboglu FO. Pulmonary function and airway hyperresponsiveness in adults with sickle cell disease. Lung 2009;187:195-200.

9. Strunk RC, Brown MS, Boyd JH, Bates P, Field JJ, DeBaun MR. Methacholine challenge in children with sickle cell disease: a case series. Pediatric pulmonology 2008;43:924-9.

10. Field JJ, Stocks J, Kirkham FJ, et al. Airway hyperresponsiveness in children with sickle cell anemia. Chest 2011;139:563-8.

11. Koumbourlis AC, Hurlet-Jensen A, Bye MR. Lung function in infants with sickle cell disease. Pediatr Pulmonol 1997;24:277-81.

12. Sylvester KP, Patey RA, Rafferty GF, Rees D, Thein SL, Greenough A. Airway hyperresponsiveness and acute chest syndrome in children with sickle cell anemia. Pediatric pulmonology 2007;42:272-6.

13. Bryant R. Asthma in the pediatric sickle cell patient with acute chest syndrome. Journal of pediatric health care : official publication of National Association of Pediatric Nurse Associates \& Practitioners $2005 ; 19: 157-62$. 
14. Nordness ME, Lynn J, Zacharisen MC, Scott PJ, Kelly KJ. Asthma is a risk factor for acute chest syndrome and cerebral vascular accidents in children with sickle cell disease. Clinical and molecular allergy : CMA 2005;3:2.

15. Boyd JH, Macklin EA, Strunk RC, DeBaun MR. Asthma is associated with increased mortality in individuals with sickle cell anemia. Haematologica 2007;92:1115-8.

16. Boyd JH, Macklin EA, Strunk RC, DeBaun MR. Asthma is associated with acute chest syndrome and pain in children with sickle cell anemia. Blood 2006;108:2923-7.

17. Knight-Madden JM, Forrester TS, Lewis NA, Greenough A. Asthma in children with sickle cell disease and its association with acute chest syndrome. Thorax 2005;60:206-10.

18. Vij R, Machado RF. Pulmonary complications of hemoglobinopathies. Chest 2010;138:973-83.

19. de Jong PA, Nakano Y, Lequin MH, et al. Progressive damage on high resolution computed tomography despite stable lung function in cystic fibrosis. European Respiratory Journal 2004;23:93-7.

20. Brody AS, Klein JS, Molina PL, Quan J, Bean JA, Wilmott RW. High-resolution computed tomography in young patients with cystic fibrosis: distribution of abnormalities and correlation with pulmonary function tests. The Journal of pediatrics 2004;145:32-8.

21. Amin R, Subbarao P, Jabar A, et al. Hypertonic saline improves the LCI in paediatric patients with CF with normal lung function. Thorax 2010;65:379-83.

22. Amin R, Subbarao P, Lou W, et al. The effect of dornase alfa on ventilation inhomogeneity in patients with cystic fibrosis. The European respiratory journal 2011;37:806-12.

23. Gustafsson PM, De Jong PA, Tiddens HA, Lindblad A. Multiple-breath inert gas washout and spirometry versus structural lung disease in cystic fibrosis. Thorax 2008;63:129-34.

24. Ellemunter H, Fuchs SI, Unsinn KM, et al. Sensitivity of Lung Clearance Index and chest computed tomography in early CF lung disease. Respiratory medicine 2010;104:1834-42.

25. Lopes AJ, Marinho CL, Alves UD, et al. Relationship between ventilation heterogeneity and exercise intolerance in adults with sickle cell anemia. Brazilian journal of medical and biological research = Revista brasileira de pesquisas medicas e biologicas / Sociedade Brasileira de Biofisica [et al] 2017;50:e6512.

26. MacLean JE, Atenafu E, Kirby-Allen M, et al. Longitudinal decline in lung volume in a population of children with sickle cell disease. Am J Respir Crit Care Med 2008;178:1055-9.

27. Robinson PD, Latzin P, Verbanck S, et al. Consensus statement for inert gas washout measurement using multiple- and single- breath tests. The European respiratory journal 2013;41:507-22.

28. Horsley A. Lung clearance index in the assessment of airways disease. Respiratory medicine 2009;103:7939 .

29. Standardization of Spirometry, 1994 Update. American Thoracic Society. American journal of respiratory and critical care medicine 1995;152:1107-36.

30. American Thoracic Society. Single-breath carbon monoxide diffusing capacity (transfer factor). Recommendations for a standard technique-1995 update. American journal of respiratory and critical care medicine 1995;152:2185-98.

31. Culver BH, Graham BL, Coates AL, et al. Recommendations for a Standardized Pulmonary Function Report. An Official American Thoracic Society Technical Statement. American journal of respiratory and critical care medicine 2017;196:1463-72.

32. Zwitserloot A, Fuchs SI, Muller C, Bisdorf K, Gappa M. Clinical application of inert gas Multiple Breath Washout in children and adolescents with asthma. Respiratory medicine 2014;108:1254-9. 
33. Halphen I, Elie C, Brousse V, et al. Severe nocturnal and postexercise hypoxia in children and adolescents with sickle cell disease. PLoS One 2014;9:e97462.

34. Hargrave DR, Wade A, Evans JP, Hewes DK, Kirkham FJ. Nocturnal oxygen saturation and painful sickle cell crises in children. Blood 2003;101:846-8.

35. Cohen RT, Strunk RC, Rodeghier M, et al. Pattern of Lung Function Is Not Associated with Prior or Future Morbidity in Children with Sickle Cell Anemia. Ann Am Thorac Soc 2016;13:1314-23.

36. Pritchard KA, Jr., Feroah TR, Nandedkar SD, et al. Effects of experimental asthma on inflammation and lung mechanics in sickle cell mice. Am J Respir Cell Mol Biol 2012;46:389-96.

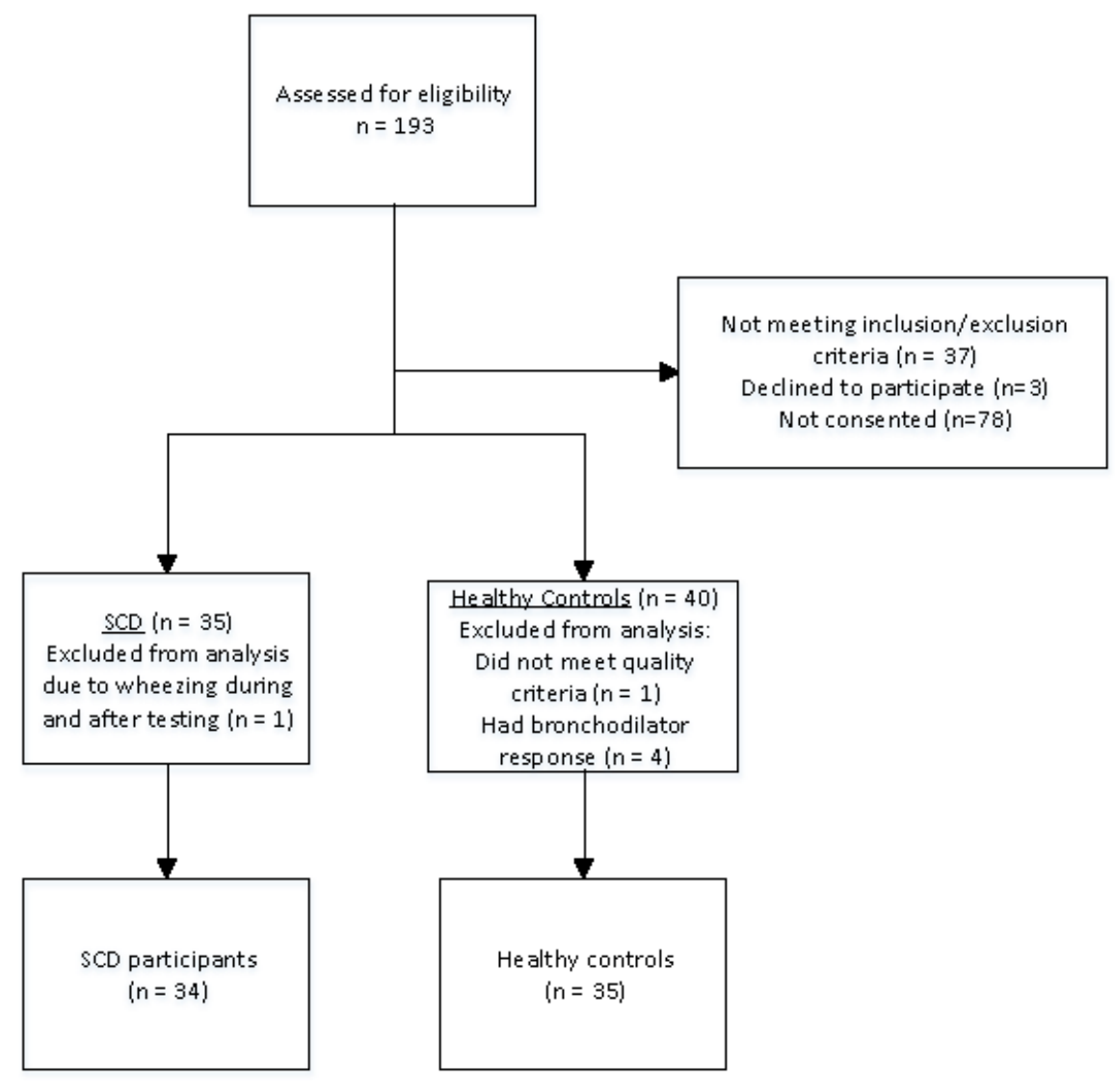




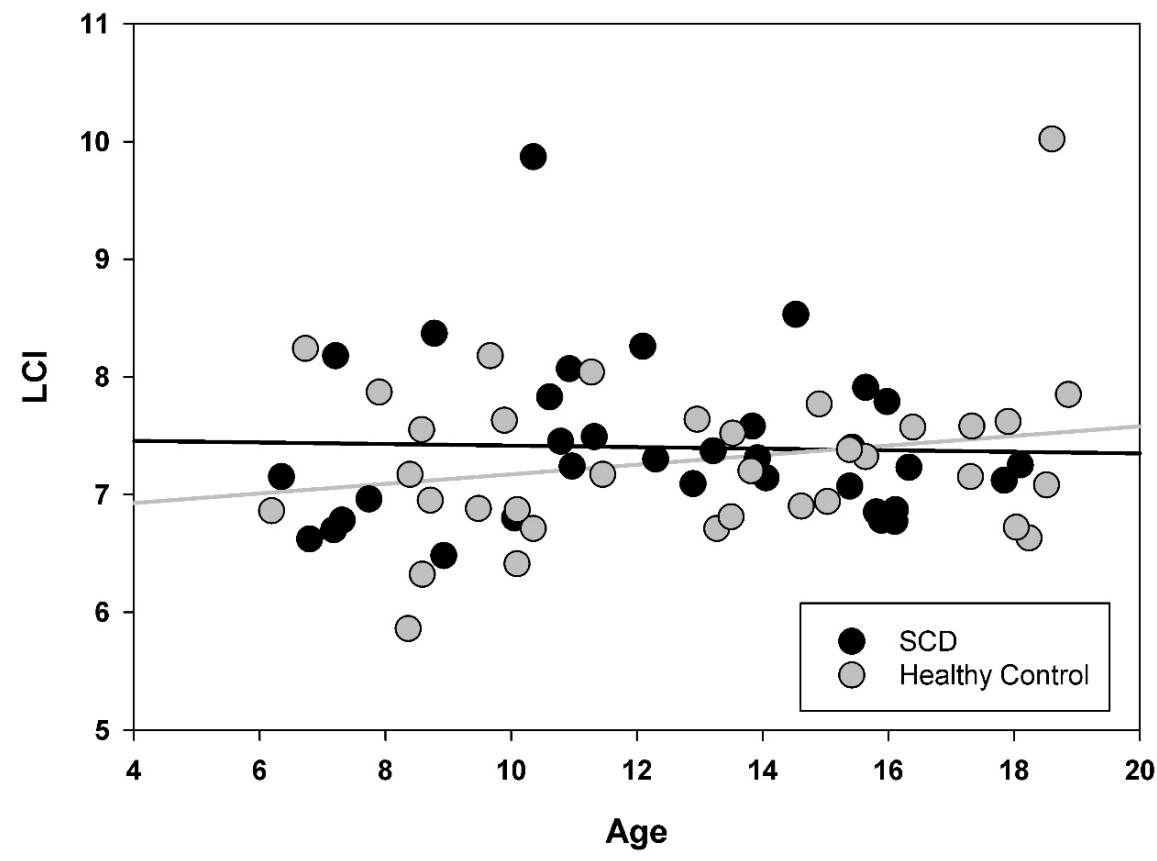

\section{Hosted file}

Tables.docx available at https://authorea.com/users/340661/articles/467695-lung-clearanceindex-in-children-with-sickle-cell-disease 